\title{
Size Matters: Origin of Binomial Scaling in Nuclear Fragmentation Experiments
}

\author{
Wolfgang Bauer and Scott Pratt \\ Department of Physics and Astronomy and \\ National Superconducting Cyclotron Laboratory, \\ Michigan State University, East Lansing, MI $48824 \quad$ USA
}

(June 15, 2021)

\begin{abstract}
The relationship between measured transverse energy, total charge recovered in the detector, and size of the emitting system is investigated. Using only very simple assumptions, we are able to reproduce the observed binomial emission probabilities and their dependences on the transverse energy.
\end{abstract}

Typeset using REVTEX 
During the last decade, evidence has been mounting that nuclear matter undergoes a phase transition in the nuclear fragmentation process. From general consideration regarding the elementary nucleon-nucleon interaction (repulsive at short and attractive at intermediate distances), we expect the nuclear phase-diagram to show a Van-der-Waals "liquid-gas" phase transition of first order, terminating in a second-order transition at the critical point.

Recent observations point to evidence of first and second order transitions. In experiments studying Au-Au collisions conducted at the GSI, a measurement of the temperature as a function of excitation energy found possible evidence for a two-phase coexistence regime [1], not unlike the scenarios predicted by statistical multifragmentation models with excluded volume [2, 3]. Other experiments conducted at the Bevalac focussed on the extraction of critical exponents from (almost) completely reconstructed $\mathrm{Au}$-fragmentation events on $\mathrm{C}$ targets, studying the dependence of the second moment of the charge distribution and size of the largest fragment as a function of the total charged particle multiplicity [4 [6]. It was shown [7,8] that these data are consistent with the second-order phase transition predicted by the nuclear percolation model [9] [12].

If one wants to gain a fundamental understanding of the fragmentation process that goes beyond simple equilibrium model descriptions of the phenomena, then a proper description of the origin and time evolution of fluctuations is essential [13 [16], in particular if one wants to understand why particular molecular dynamics codes produce fragments (or not!), and what their connections to the fundamental processes of nuclear fragmentation are 17 27.

In this light, the recent findings of Moretto et al. are all the more surprising 28 32. This group found that the probability $P_{n}$ of emitting $n$ intermediate mass fragments (IMFs) follows a binomial distribution

$$
P_{n}(m, p)=\frac{m !}{n !(m-n) !} p^{n}(1-p)^{m-n}
$$

The parameters $m$ and $p$ are related to the average and variance of the distribution.

$$
\langle n\rangle=\sum_{n=0}^{\infty} n P_{n}(m, p)=m \cdot p
$$




$$
\sigma^{2}=\sum_{n=0}^{\infty}(n-\langle n\rangle)^{2} P_{n}(m, p)=m p(1-p)
$$

This result suggest that one may interpret the parameter $p$ as the elementary probability for the emission of one fragment and the parameter $m$ as the total number of tries. This would indicate that the problem of multi-fragment emission is reducible to that of multiple one-fragment emission. The claim for reducibility and its interpretation as the consequence of a simple barrier penetration phenomenon was further strengthened by the observation that $\ln \left(p^{-1}\right)$ has a linear dependence on $1 / \sqrt{E_{t}}$, where $E_{t}$ is the total transverse energy, $E_{t}=\sum_{l} E_{k l} \sin ^{2} \theta_{l}$. Finally, the same scaling was found for different beam energies and different projectile-target combinations.

Other authors have criticized the above work, pointing our that there are different emission probabilities for different size IMFs, that there are problems in the transformation between the total transverse energy and a true thermal energy [33], and focussing on autocorrelations between the number of IMFs and the transverse energy [34]. (See also the replies to these criticisms in refs. 32,35.)

In the present note we add to this discussion by showing how binomial distributions arise naturally from finite size effects. In particular, we focus on the dependence of the experimentally recovered charge as a function of the measured transverse energy. We then demonstrate why the dependence of the binomial parameter $p$ on the total transverse energy arises.

We begin our study by generating power-law distributed random fragmentation events. This is accomplished by determining the charge of individual fragments with a probability distribution proportional to $Z^{-\tau}$, where $Z$ is the fragment charge, and $\tau$ is the power-law exponent. For definiteness, we wish to generate events with exactly $Z_{\text {sys }}$ charges. If an event has less than $Z_{\text {sys }}$ charges, we add another fragment; if it has more than $Z_{\text {sys }}$ charges, we throw it out. For an infinite system, we would expect the multiplicity distributions for individual fragments of a given $Z$ to follow a Poisson distribution,

$$
Q_{n}(\lambda)=\frac{\lambda^{n} \exp (-\lambda)}{n !} \quad \text { with }: \quad \lambda=\langle n\rangle=\sigma^{2}
$$


And since the combined probability distribution of two Poisson-distributed variables is again a Poissonian,

$$
Q_{n}\left(\lambda_{1}\right) \otimes Q_{n}\left(\lambda_{2}\right) \equiv \sum_{i=0}^{n} Q_{i}\left(\lambda_{1}\right) \cdot Q_{n-i}\left(\lambda_{2}\right)=Q_{n}\left(\lambda_{1}+\lambda_{2}\right)
$$

we would expect that the multiplicity distribution of the total number of intermediate mass fragments (IMFs) is also Poissonian.

The individual probability distributions for IMFs, however, cannot be exactly Poissonian, because the tails of the distributions are cut off due to the finite size of the emitting system. Thus the probability distributions in our simulation are closer to a binomial distribution with rather large values of $m$ and small values of $p$. (When $m \rightarrow \infty, p \rightarrow 0$ such that $m p=$ const., we obtain a Poissonian as the limit of a binomial distribution.) Typical values of $p$ we find for the probability distributions of our individual fragments are $\leq 3 \cdot 10^{-2}$ for a system of 100 total charges. These small values of $p$ imply that the probability distributions are very close to a Poisson distribution.

We now ask what the combined probability distribution for fragments charges in the interval 3 to $k, k=4,5, \ldots, 30$ is. (If we use $k=20$, this corresponds to the usual definition of IMFs.) We find numerically that to very good approximation this distribution is again a binomial distribution, for all values of $k$. The binomial parameters $p_{k}$ and $m_{k}$ have a monotonical behavior as a function of $k: p_{k}$ rises monotonically until it saturates at $k=Z_{\text {sys }}$, and $m_{k}$ falls monotonically. This results directly from the mathematical fact that the mean of the combined probability distribution is the sum of the mean values of the individual distributions, but the variance is always smaller than the sum of the variances for the folding of binomial distributions.

In figure 1 , we show the behavior of the parameters $p_{k}$ and $m_{k}$ of the combined probability distributions as a function of $k$, the upper limit charge for the folding procedure, for different values of $Z_{\text {sys }}$. For each value of $Z_{\text {sys }}$, we generated $10^{4}$ events. This figure already contains the essential key to understanding the patterns observed by Moretto and collaborators. We can clearly see that as we include more and more fragments in the definition of IMFs the 
extracted values of the binomial parameter $p_{k}$ increase, and those for $m_{k}$ decrease. We can also see that $p_{3}\left(=p_{k}(k=3)\right)$ decreases as we increase the total charge of the fragmenting system. This is expected: the larger the total available charge, the closer the probability distributions of individual fragments (in this case $Z=3$ ) will be to the Poissonian limit. It is essential to note that the values of $p_{k}$ for each system size saturate at $k=Z_{\text {sys }}$. This is obvious, because we cannot have IMFs larger than the total charge available. But this obvious fact has an interesting consequence: The smaller the system size, the fewer the terms that can contribute to the construction of the asymptotic value of $p_{k}$, and the lower the asymptotic value of $p_{k}$.

This fact, combined with the dependence of $Z_{\text {sys }}$ on the transverse energy, already is the explanation for the scaling observed by Moretto et al.

What is the dependence of $Z_{\text {sys }}$ on the transverse energy, $E_{t}$, in the experiments of Moretto et al.? This is shown in fig. 2 for the reaction $\mathrm{Kr}+\mathrm{Au}$ at $55 \mathrm{AMeV}$ [36, 37. The filled plot symbols show the mean $Z_{\text {sys }}$ for each value of the $E_{t}$, and the error bars give the width (standard deviation) of the distribution. The dominant feature of this figure is the linear rise of the mean value of $Z_{\text {sys }}$ with $E_{t}$,

$$
\left\langle Z_{\mathrm{sys}}\left(E_{t}\right)\right\rangle \approx 2+0.092 E_{t} / \mathrm{MeV}
$$

for values of $E_{t}$ less than $0.7 \mathrm{GeV}$, and the saturation of $Z_{\text {sys }}$ for larger values.

The width of the $Z_{\text {sys }}\left(E_{T}\right)$-distribution is significant, on the order of 10 units of charge. If we wish to construct the probability distributions of intermediate mass fragments by using our knowledge of the dependence of the binomial parameters $p$ and $m$ on the system size (fig. 1), and the dependence of the system size on transverse energy, we have to integrate over the experimentally measured width of the $Z_{\text {sys }}\left(E_{T}\right)$-distribution. The resulting values of $p$ for the integrated distributions are shown as a function of $E_{t}$ in fig. 3 . We have run three different calculations, using three different values of the exponent $\tau$ in our fragment production probability distributions, $p(Z) \propto Z^{-\tau}$. In this figure, we display the results in the same way that Moretto et al. have done. One can already see that there is qualitative 
agreement with the tendencies observed by the Moretto-group: for all values of $\tau$, we observe an approximately linear rise of $\ln p^{-1}$ with $1 / \sqrt{E_{t}}$. This, however, is not the consequence of some kind of thermal scaling. Instead, it is purely a consequence of the variation of the size of the emitting system as a function of the transverse energy, and with it a change in the effective parameter $p$ in the binomial probability distribution.

The projectile and target masses only enter into our consideration as upper cutoffs for the possible maximum values for $Z_{\text {sys }}$, and with it the upper values of $E_{t}$. The experimentally found functional dependence of $Z_{\text {sys }}$ on $E_{t}$ that we show in fig. 2 for the system $\mathrm{Kr}+\mathrm{Au}$ is basically the same for all target-projectile combinations and beam energies below approximately $100 A \mathrm{MeV}$ (for higher beam energies, we have sizeable radial flow contributions to the transverse energy). Transverse energy basically measures impact parameter, that is to say size of the emitting system; and for any experiment measuring inclusive fragment distributions the results will be very much similar to the ones displayed in fig. 2. This explains the universal scaling observed by Moretto et al. without the need for invoking some deeper reason for this apparent universality; a plot of $\ln p^{-1}$ vs. $1 / \sqrt{E_{t}}$ is dominated by effects of the variation of the size of the emitting system. We should point out that our findings are not dependent on the fact that the intermediate mass fragments carry transverse energy themselves. The only correlations entering our analysis are the experimental ones between transverse energy and system size.

We can obtain more-or-less complete agreement with the experimental data, if we allow the power-law parameter $\tau$ for the fragment mass distribution to vary with impact parameter and with beam energy. This variation is a well-documented experimental fact [37 39]; the experimentally observed value of $\tau$ increases with impact parameter and therefore falls with transverse energy. If we assume

$$
\tau\left(E_{t}\right)=3.5-E_{t} /(0.5 \mathrm{GeV})
$$

then we get the result displayed in fig. 4. Our calculations are represented by the plot symbols. The error bars are statistical and computed on the basis of $2 \times 10^{4}$ events for 
each point. The solid line is a fit to the experimental results of Moretto et al. As one can see, there is very good agreement. Assuming other functional dependences of $\tau$ of $E_{t}$ may even yield better results. This agreement, however, is not quite as relevant as the main message we wish to impress on the reader: The universal scaling of $\ln p^{-1}$ vs. $1 / \sqrt{E_{t}}$ is almost exclusively due to the finite size of the system emitting the fragments and the dependence of the measured value of the transverse energy on that size.

Even though the main message of the present note is that the $E_{t}$-dependence of the extracted binomial parameters of the fragment multiplicity distributions can be explained rather straightforwardly, we do not wish to convey the message that there is no interesting information that one can extract from this type of analysis. For instance, the effects of varying system size could be eliminated with utilization of completely reconstructed fragmentation events. For these types of events, percolation models predict a transition between sub- and super-Poissonian fluctuations near the percolation threshold [16]. This type of behavior is not expected in a sequential model. Once the kind of correlations discussed by us above are removed, then this type of fluctuations analysis should yield insightful information about the character of the nuclear fragmentation phase transition.

\section{ACKNOWLEDGMENTS}

This work was supported by the National Science Foundation, grant PHY-9605207. 


\section{REFERENCES}

[1] J. Pochodzalla et al., Phys. Rev. Lett. 75, 1040 (1995); J. Pochodzalla, Prog. Part. Nucl. Phys. 39, 443 (1997).

[2] D.H.E. Gross, Rep. Prog. Phys. 53, 605 (1990).

[3] J.P. Bondorf, A.S. Botvina, A.S. Iljinov, I.N. Mishustin and K. Sneppen, Phys. Rep. 257, 133 (1995).

[4] M.L. Gilkes et al., Phys. Rev. Lett. 73, 1590 (1994).

[5] J.B. Elliott et al., Phys. Rev. C 49, 3185 (1994).

[6] H.G. Ritter et al., Nucl. Phys. A 583, 491c (1995).

[7] W. Bauer and W.A. Friedman, Phys. Rev. Lett. 75, 767 (1995).

[8] W. Bauer and A. Botvina, Phys. Rev. C 52, R1760 (1995); Phys. Rev. C 55, 546 (1997).

[9] W. Bauer et al., Phys. Lett. B150, 53 (1985).

[10] W. Bauer et al., Nucl. Phys. A452, 699 (1996).

[11] W. Bauer, Phys. Rev. C 38, 1297 (1988).

[12] X. Campi, J. Phys. A 19, L917 (1986).

[13] J. Randrup and S. Koonin, Nucl. Phys. A 356, 321 (1981).

[14] W. Bauer, G.F. Bertsch, and S. Das Gupta, Phys. Rev. Lett. 58, 863 (1987).

[15] Ph. Chomaz, Ann. Phys. France 21, 669 (1996); G.F. Burgio, Ph. Chomaz and J. Randrup, Phys. Rev. Lett. 69, 885 (1992).

[16] T. Gharib, W. Bauer, and S. Pratt, submitted to Phys. Lett. B; Michigan State University preprint, Los Alamos preprint archive nucl-th/9808065.

[17] J. Aichelin and H. Stöcker, Phys. Lett. B176, 14 (1986). 
[18] D.H. Boal and J.N. Glosli, Phys. Rev. C 38, 2621 (1988).

[19] H. Feldmeier, Nucl. Phys. A515, 147 (1990).

[20] J. Aichelin, Phys. Rep. 202, 233 (1991).

[21] A. Ono et al., Prog. of Theoret. Phys. 87, 1185 (1992).

[22] A. Ono et al., Phys. Rev. Lett. 68, 2898 (1992).

[23] V. Latora et al., Phys. Rev. Lett. 73, 1765 (1994).

[24] S. Pratt et al., Phys. Lett. B349, 261 (1995).

[25] H. Feldmeier and J. Schnack, Prog. Part. Nucl. Phys. 39, 393 (1997).

[26] A. Ohnishi and J. Randrup, Phys. Lett. B 394, 260 (1997).

[27] D. Kiderlen and P. Danielewicz, Nucl. Phys. A 620, 46 (1997).

[28] L.G. Moretto et al., Phys. Rev. Lett. 71, 3935 (1993).

[29] L.G. Moretto et al., Phys. Rev. Lett. 74, 1530 (1995).

[30] L. Phair et al., Phys. Rev. Lett. 77, 822 (1996).

[31] L.G. Moretto et al., Phys. Rep. 287, 249 (1997).

[32] L. Beauleau et al., in Proceedings of the 14th Winter Workshop on Nuclear Dynamics, ed.: W. Bauer and H.G. Ritter (Plenum, New York, 1998), to be published.

[33] J. Toke, D.K. Agnihotri, B. Djerroud, W. Skulski and W.U. Schroeder, Phys. Rev. C 56, R1686 (1997).

[34] M.B. Tsang and P. Danielewicz, Phys. Rev. Lett. 80, 1178 (1998).

[35] L.G. Moretto, L. Bealieu, L. Phair, and G.J. Wozniak, Los Alamos preprint archive nucl-ex/9709001 (1997). 
[36] M.B. Tsang, private communication.

[37] C. Williams et al., Phys. Rev. C 55, R2132 (1997).

[38] T. Li et al., Phys. Rev. Lett. 70, 1924 (1993).

[39] T. Li et al., Phys. Rev. C 49, 1630 (1994). 


\section{FIGURES}

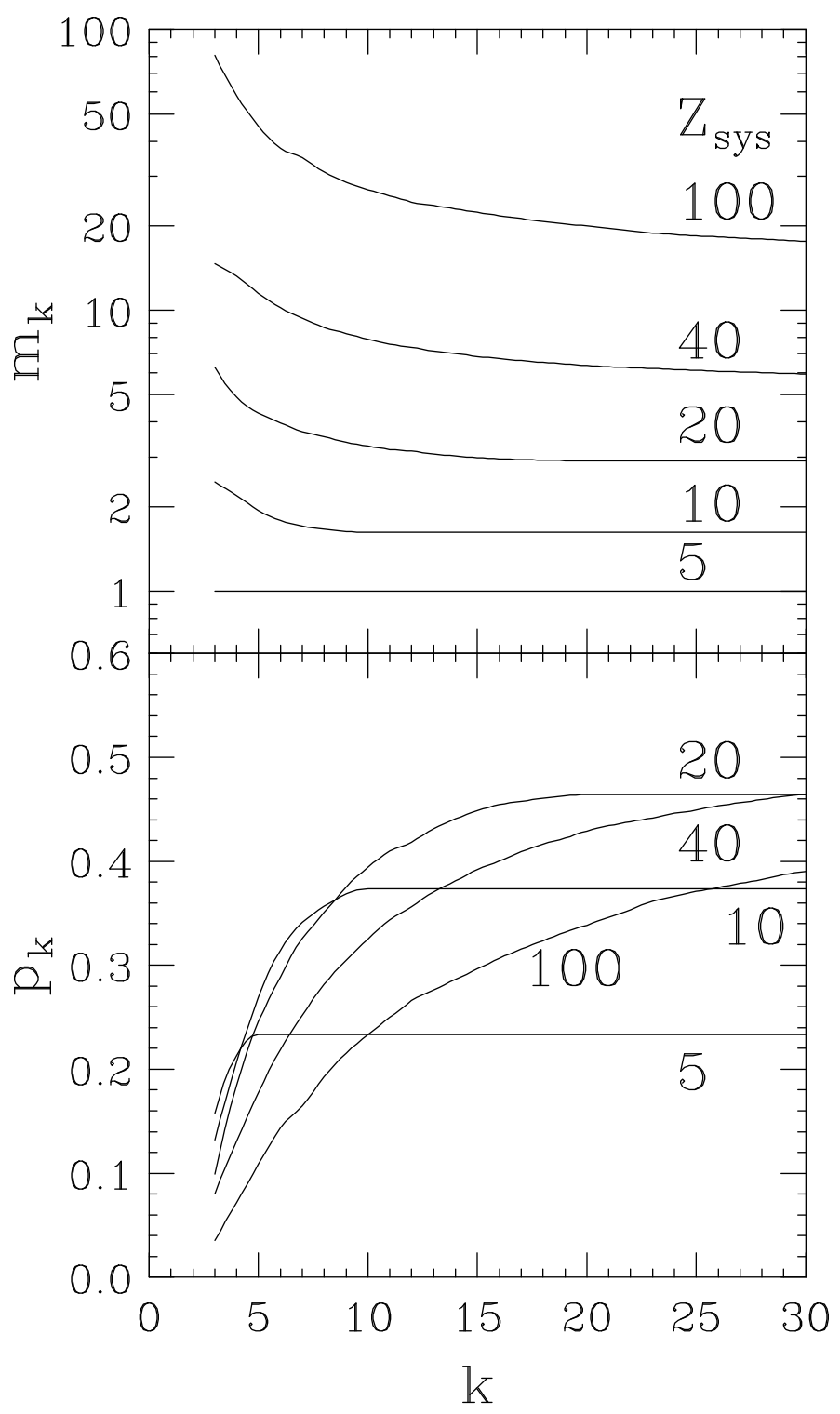

FIG. 1. Dependence of the binomial fit parameters $p_{k}$ and $m_{k}$ of the probability distributions for intermediate mass fragments from charge $Z=3$ to $Z=k$ on the upper summation limit $k$, for different total charges of the fragmenting system, $Z_{\text {sys }}$. 


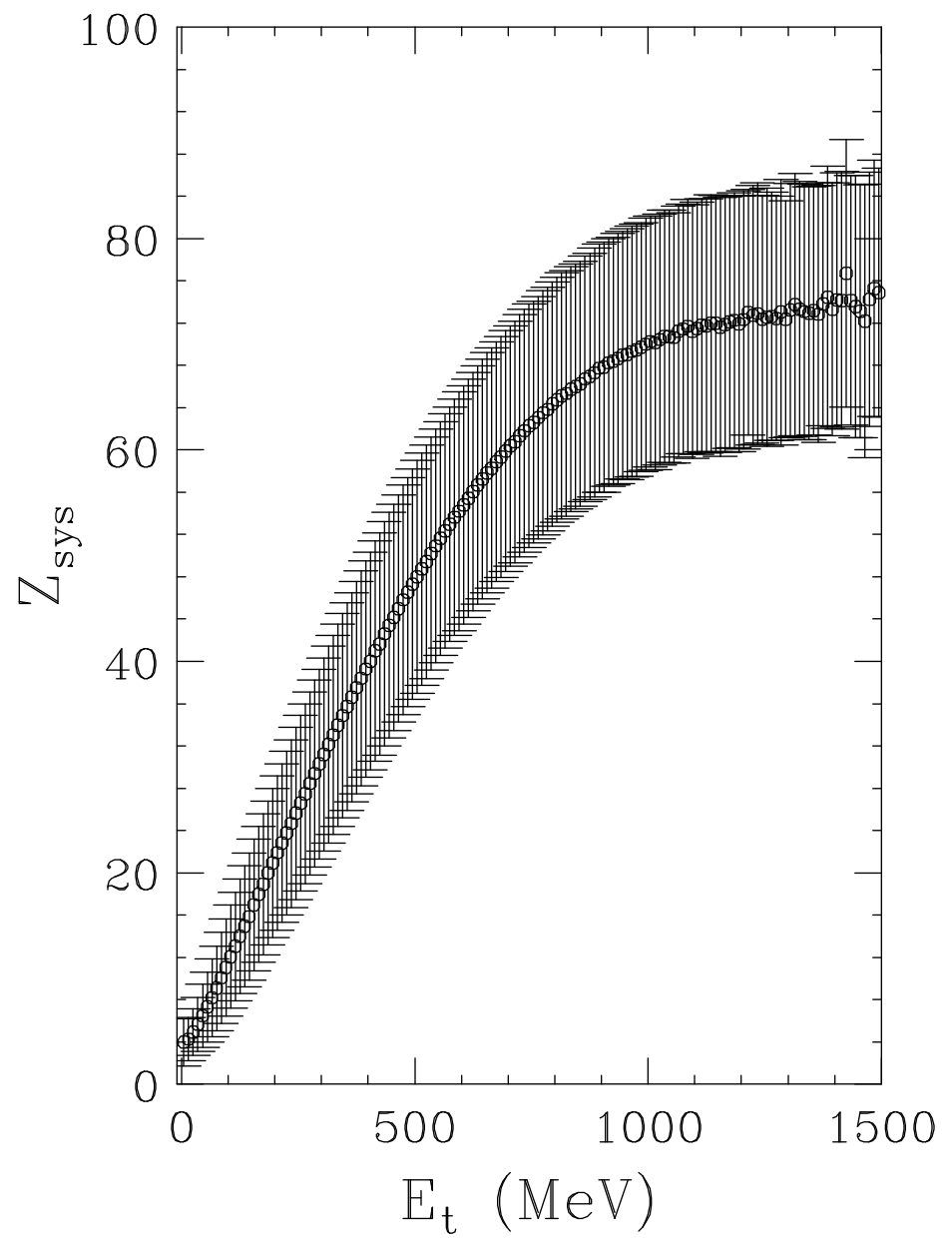

FIG. 2. Dependence of the total charges of detected in a fragmentation event, $Z_{\text {sys }}$, on the total transverse energy, $E_{t}$, detected in the experiment $55 \mathrm{AMeV} \mathrm{Kr}+\mathrm{Au}$ [36]. The error bars indicate the width (standard deviation) of the distribution on an event-by-event basis. 


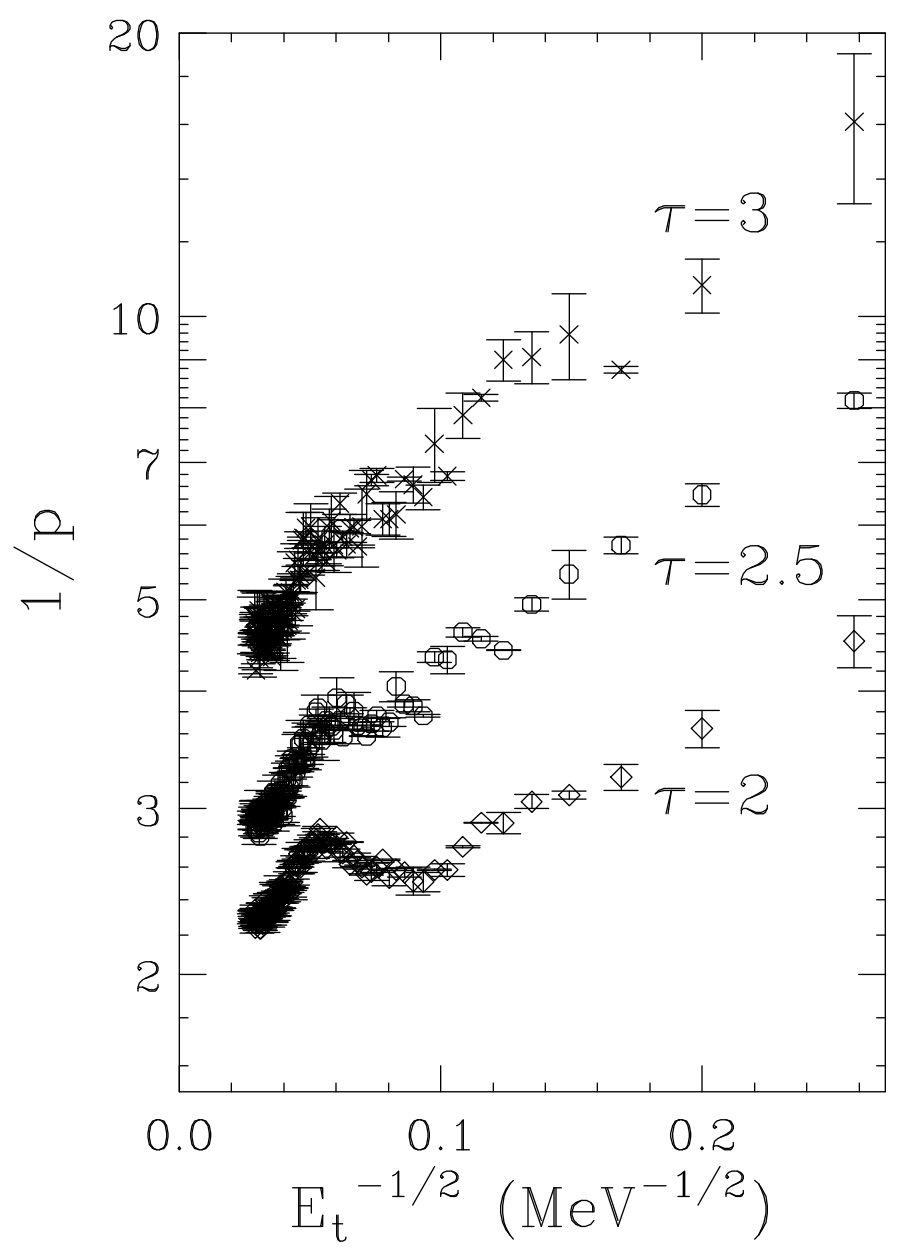

FIG. 3. Dependence of the binomial parameter $p$ of the IMF distribution on the transverse energy for three different values of $\tau$. 


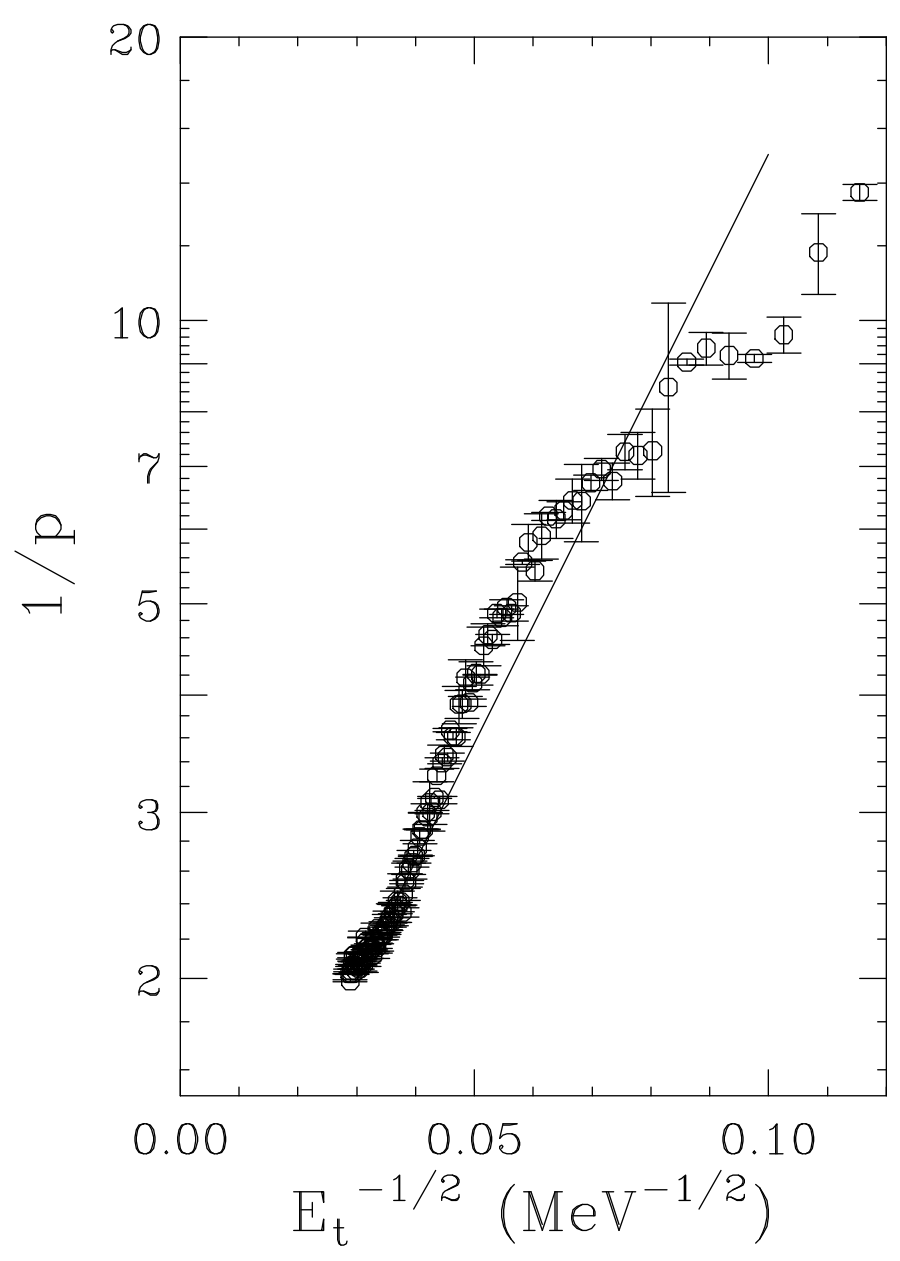

FIG. 4. Dependence of the binomial parameter $p$ of the IMF distribution on the transverse energy, assuming that the effective power $\tau$ of the fragment probability increases linearly with transverse energy. Plot symbols with error bars represent our calculations, the solid line is a fit to the experimental data of Moretto and collaborators. 